Relations industrielles

Industrial Relations

\title{
Andrew J. DUBRIN : Foundations of Organizational Behavior : An Applied Perspective. Englewood Cliffs, Prentice-Hall, 1984, 500 pp., ISBN 0-13-329367-X
}

\section{Laurent Bélanger}

\section{Volume 39, numéro 4, 1984}

URI : https://id.erudit.org/iderudit/050094ar

DOI : https://doi.org/10.7202/050094ar

Aller au sommaire du numéro

Éditeur(s)

Département des relations industrielles de l'Université Laval

ISSN

0034-379X (imprimé)

1703-8138 (numérique)

Découvrir la revue

Citer ce compte rendu

Bélanger, L. (1984). Compte rendu de [Andrew J. DUBRIN : Foundations of Organizational Behavior : An Applied Perspective. Englewood Cliffs, Prentice-Hall, 1984, 500 pp., ISBN 0-13-329367-X]. Relations industrielles / Industrial Relations, 39(4), 805-806. https://doi.org/10.7202/050094ar

Tous droits réservés (C) Département des relations industrielles de l'Université Laval, 1984
Ce document est protégé par la loi sur le droit d'auteur. L’utilisation des services d'Érudit (y compris la reproduction) est assujettie à sa politique d'utilisation que vous pouvez consulter en ligne.

https://apropos.erudit.org/fr/usagers/politique-dutilisation/ 
personnel. «Si la guerre est trop importante pour la laisser aux mains des généraux, rappelle-t-il, il en est de même de la gestion du personnel qui ne doit pas être laissée aux seuls agents de personnel!» D'ailleurs ce livre s'adresse d'abord et avant tout aux gestionnaires. L'auteur leur rappelle constamment que «the name of the game in business is personnel» ou que "Personnel has become the focus of the highest level thinking and policymaking in business».

Sloane traite dans son livre, de façon fort pratique, des problèmes suivants: Comment intégrer son personnel? Comment lui donner une formation initiale adéquate, le recycler? Comment aborder le développement des cadres? Leur plan de carrière? Comment évaluer son personnel de façon à améliorer non seulement leur rendement mais leur motivation et leur satisfaction? Toutes ces questions, et bien d'autres, sont soulevées avec des exemples, des techniques, des approches nouvelles qui font de ce «textbook» un outil fort utile pour tous les gestionnaires.

Deux sections de trois chapitres chacun et de plus de 200 pages abordent des thèmes qui sont souvent escamotés ou tout simplement ignorés dans des ouvrages du même genre. Ces thèmes sont ceux des mol.. de rémunérations, des différents incitatifs monétaires, des avantages sociaux, des systèmes de pensions, de la santé et de la sécurité au travail et enfin de l'attitude que doit avoir le gestionnaire face à la discipline au travail. Sloane ne minimise aucun de ces thèmes qui ont pris au cours des années une importance sans cesse accrue. Il n'est que de signaler l'ampleur qu'ont pris les avantages sociaux avec le temps. De $1 \%$ qu'ils étaient en 1929 , ils représentent plus de $35 \%$ de la rémunération aujourd'hui, avec une croissance annuelle de $1 \%$ ! D'autre part, qui aurait prévu, il y a à peine dix ans, la proportion qu'occupent et qu'occuperont en l'an 2000 , les retraités. Qu'en sera-t-il de leurs pensions? Sloane aborde ce theme avec beaucoup de lucidité et propose des solutions qui ont déjà été éprouvées dans certaines organisations.

Enfin, dans une dernière section, l'auteur présente le thème des relations du travail: une vue d'ensemble de la question, l'évolution des relations patronalesouvrières, la négociation et l'administration des conventions collectives. Cette partie, bien qu'intéressante, ne présente pas le même intérêt que les précédentes. Peut-être, est-ce dû au fait que l'évolution du monde du travail et les luttes syndicales ont pris aux Etats-Unis une ampleur et des tendances différentes des nôtres.

Arthur Sloane termine son ouvrage en invoquant quelques-uns des défis que devront affronter les gestionnaires en matière de gestion du personnel: le respect de la vie privée et des droits des employés, la place des femmes au travail, les burnouts, l'introduction de nouvelles formes et de nouvelles conditions de travail, l'approche devant l'implantation des nouvelles technologies.

En somme un «textbook» de plus de 600 pages qui fait le point sur la gestion des ressources humaines. Un livre qui rend caducs plusieurs de ceux qui ont été écrits récemment. Un manuel qui présente des exemples, des techniques nouvelles, des approches qui ont été utilisées avec succès dans certaines entreprises. Enfin un livre qui, par sa forme, s'adresse non seulement aux étudiants mais aussi à ceux qui leur enseignent!

Paul TESSIER

Ecole nationale d'administration publique Québec

Foundations of Organizational Behavior: An Applied Perspective, by Andrew J. Dubrin, Englewood Cliffs, Prentice-Hall In., 1984, 500 pp., ISBN-0-13-329367-x

Cet ouvrage s'adresse presqu'uniquement aux étudiants de premier cycle universitaire dans les facultés de sciences de l'administration et dans les départements de relations industrielles. Par conséquent, il ne faut pas se surprendre de n'y retrouver que les thèmes largement connus qui constituent le noyau des sciences du comportement formé des éclairages particuliers de la psychologie, de la sociologie et de l'anthropologie appliquées aux organisations de travail. 
Ces thèmes sont regroupés sous quatre grandes rubriques qui constituent les quatre parties de l'ouvrage. Dans la partie introductive, l'auteur tente de retracer l'évolution du domaine des sciences du comportement depuis les recherches et les enseignements de l'École des relations humaines jusqu'aux études récentes traitant du stress au travail et du phénomène de l'épuisement (burn out). Cette introduction fait également état des méthodes de recherche propres à l'étude du comportement humain en faisant ressortir la manière dont les connaissances se sont accumulées dans ce domaine.

Sans vouloir imposer, voire même suggérer, un cadre de référence ou une problématique, l'auteur s'en tient au cours de la rédaction des parties principales, à un regroupement des connaissances en retenant les trois pôles suivants: d'abord, l'étude des différences individuelles (perceptions, attitudes, valeurs, motivations, satisfaction, stress, créativité) et leur impact sur le comportement; ensuite, l'étude des structures et $\mathrm{du}$ fonctionnement des groupes de travail; enfin, dans une perspective macro-organisationnelle, l'étude des structures et du climat organisationnels pour terminer avec une présentation des concepts et de la démarche propre au développement des organisations ou à l'amélioration de la qualité au niveau travail.

Ce n'est pas tellement par son contenu que cet ouvrage se distingue d'une foule d'autres déjà publiés dans le même domaine, mais bien par une certaine préoccupation pédagogique que l'auteur manifeste.

Il s'en tient au vocabulaire qui se situe a mi-chemin entre les concepts abstraits utilisés par les chercheurs ou les académiciens et les termes quotidiens peu précis qu'on emploi pour décrire des phénomènes de comportement au niveau de l'individu, du groupe et de l'organisation de travail.

Chaque chapitre débute par une présentation des objectifs à atteindre au plan de l'acquisition des connaissances. Suit un incident qui illustre les enseignements véhiculés dans le chapitre et la pertinence de ces enseignements dans la pratique. Chaque chapitre se termine par la présentation d'un problème à résoudre et d'une liste de questions permettant un retour sur ce qui a été exposé. À la fin de l'ouvrage, on retrouve un lexique des termes-clef utilisés dans le domaine.

Les dirigeants d'entreprise de même que les professionnels de la gestion des ressources humaines n'y verront encore qu'un recueil d'observations ou de lignes de conduite très proches du sens commun. Bien entendu, la connaissance $\mathrm{du}$ comportement humain n'échappe pas au sens commun ou à la sagesse populaire, heureusement...

Cependant, pour aller au-delà du sens commun, ne serait-ce que pour accéder à ce que j'appellerais un certain sens commun affiné (enlighted common sense), la lecture de ce type d'ouvrage aide énormément. Elle permet une meilleure connaissance et une compréhension des phénomènes humains, de sorte que toute décision même minime, dans le domaine de l'utilisation des ressources humaines, s'en trouve affectée.

Laurent BÉLANGER

Université Laval

L'entretien d'appréciation du personnel: pratiques en question, par Jacques Piveteau, Toulouse, Editions Erès, 1981, 207 pp., ISBN 2-86586-002-7

Publié en France, il y a déjà quelques années, il n'en demeure pas moins que le sujet traité par Piveteau est d'actualité et très pertinent aux lecteurs québécois et canadiens.

Une enquête récente (Politiques et pratiques en évaluation de la performance du personnel dans les organisations au Québec, par R. Thériault et J.Y. Le Louarn, Montréal, Association des professionnels en ressources humaines du Québec, 1984) sur les politiques et pratiques en évaluation de la performance du personnel révèle que plus de cinquante pour cent des organisations au Québec ont modifié leurs systèmes d'appréciation de la performance du personnel, au cours de la dernière année, ou sont en train de le faire. Trente pour cent se proposent de le faire d'ici 